\title{
Simultaneous in situ X-ray Diffraction and Calorimetric Studies as a Tool to Evaluate Gas Adsorption in Microporous Materials
}

William R. Woerner, ${ }^{1}$ Anna M. Plonka, ${ }^{1, *}$ Xianyin Chen, ${ }^{2}$ Debasis Banerjee, ${ }^{3}$ Praveen K. Thallapally, ${ }^{3}$ and John B. Parise ${ }^{1,2, *}$

${ }^{1}$ Department of Geosciences, Stony Brook University, Stony Brook, New York 11794, USA

${ }^{2}$ Department of Chemistry, Stony Brook University, Stony Brook, New York 11794, USA

${ }^{3}$ Fundamental \& Computational Sciences Directorate, Pacific Northwest National Laboratory, Richland, Washington 99354, USA

*anna.plonka@yu.edu; john.parise@,stonybrook.edu 


\section{Design of the XRD-DSC system}

The XRD-DSC system is composed of a Rigaku Ultima IV diffractometer (CuK $\alpha$ radiation with a D/TeX high speed linear position sensitive detector), a Rigaku XRD-DSC stage, Rigaku HUM1 humidity generator, ULVAC vacuum pump ( $5 \times 10^{-4}$ Torr), a vacuum manifold, and a custom built humid atmosphere swing chamber (Fig. S5). The humid atmosphere swing chamber (HASC) was necessary to buffer between the humidity generator and a XRD-DSC stage so that both humid atmosphere and vacuum swings could be performed.

The system is organized such that the desired gas flows from the selection manifold through a flow meter and into the humidity generator where mass flow controllers balance the mixing of wet and dry gas streams (Fig. S6). The humidified gas mixture then flows into the HASC where the relative humidity $(\mathrm{RH})$ of the gas stream can stabilize based on a feedback interaction between the humidity probe and generator (Fig. S7). Once the atmosphere has stabilized at the desired humidity, it is directed to the XRD-DSC stage through a needle valve. The vacuum manifold can pull vacuum directly on the XRD-DSC stage or allow gas to flow over the sample in the stage and exhaust to a fume hood.

The humidity of the atmosphere in the chamber is controlled through a feedback loop between the humidity probe and generator. The humid gas enters the chamber from the generator and can pass through to an exhaust until the atmosphere has a stable flow at the desired RH. The valve can then be switched to deliver the humid atmosphere to the sample on the XRD-DSC stage. In addition to atmosphere swings, the chamber allows for vacuum-humid atmosphere swings as the input from the generator can be closed off. Thus when the valve is opened between the XRDDSC stage (at vacuum) and the chamber, the syringe base of the HASC moves due to the pressure differential and delivers the humid atmosphere to the sample in the XRD-DSC stage. 


\section{Synthesis of the studied materials}

SBMOF-1, ZIF7 and NIMOF-74 were synthesized under solvothermal conditions using Telfonlined 23-mL Parr stainless steel autoclaves. NaX was purchased (Linde 13X).

SBMOF-1: A mixture of calcium chloride $(0.07 \mathrm{~g})$ and 4,4'-sulfonyldibenzoic acid $\left(\mathrm{H}_{2} \mathrm{sdb}\right.$, $0.19 \mathrm{~g}$ ) was dissolved in $10 \mathrm{~g}$ of ethanol and stirred for $2 \mathrm{~h}$ to achieve homegeneity. The resultant solution was heated at $180^{\circ} \mathrm{C}$ for 5 days. Colorless needle shaped crystals were recovered as products and washed with ethanol.

ZIF-7: A solid mixture of zinc nitrate tetrahydrate $\mathrm{Zn}\left(\mathrm{NO}_{3}\right)_{2}{ }_{4} \mathrm{H} 2 \mathrm{O}(0.045 \mathrm{~g})$ and benzimidazole $(0.070 \mathrm{~g})$ was dissolved in $5 \mathrm{~mL}$ of DMF and stirred for $2 \mathrm{~h}$ to achieve homogeneity. The resultant solution was heated for 3 days at $100{ }^{\circ} \mathrm{C}$ and the product was collected by filtration, washed using DMF and allowed to air-dry.

Ni MOF-74: A solid mixture of nickel nitrate hexahydrate $(0.15 \mathrm{~g})$ and 2,5-dihydroxyterephthalic acid (DHTA, $0.050 \mathrm{~g}$ ) was dissolved in $6 \mathrm{~mL}$ of DMF, $6 \mathrm{~mL}$ of ethanol and $6 \mathrm{~mL}$ of water and stirred for $2 \mathrm{~h}$ to achieve homogeneity. The resultant solution was heated for 3 days at $100{ }^{\circ} \mathrm{C}$ and the product was collected by filtration, washed using ethanol and allowed to air-dry.

\section{Thermogravimetric analysis (TGA)}

In order to determine the temperature of activation thermogravimetric analysis was performed. TGA traces for SBMOF-1, ZIF-7, Ni-MOF-74 and NaX were collected using a Netzsch 449C Jupiter instrument. Before data collection a background curve was measured with an empty alumina crucible. Next, approximately $10 \mathrm{mg}$ of the sample was placed into the alumina crucible, which is then heated to $750^{\circ} \mathrm{C}$ at a rate of $10^{\circ} \mathrm{C} / \mathrm{min}$ in a $\mathrm{N}_{2}$ atmosphere. The weight of the sample was monitored throughout the experiment.

The temperature of activation was determined as $290^{\circ} \mathrm{C}$ for SBMOF- $1,200^{\circ} \mathrm{C}$ for ZIF-7, $200^{\circ} \mathrm{C}$ for Ni-MOF-74 and $300^{\circ} \mathrm{C}$ for NaX (Figs. S9-S11) 


\section{Supplementary figures}
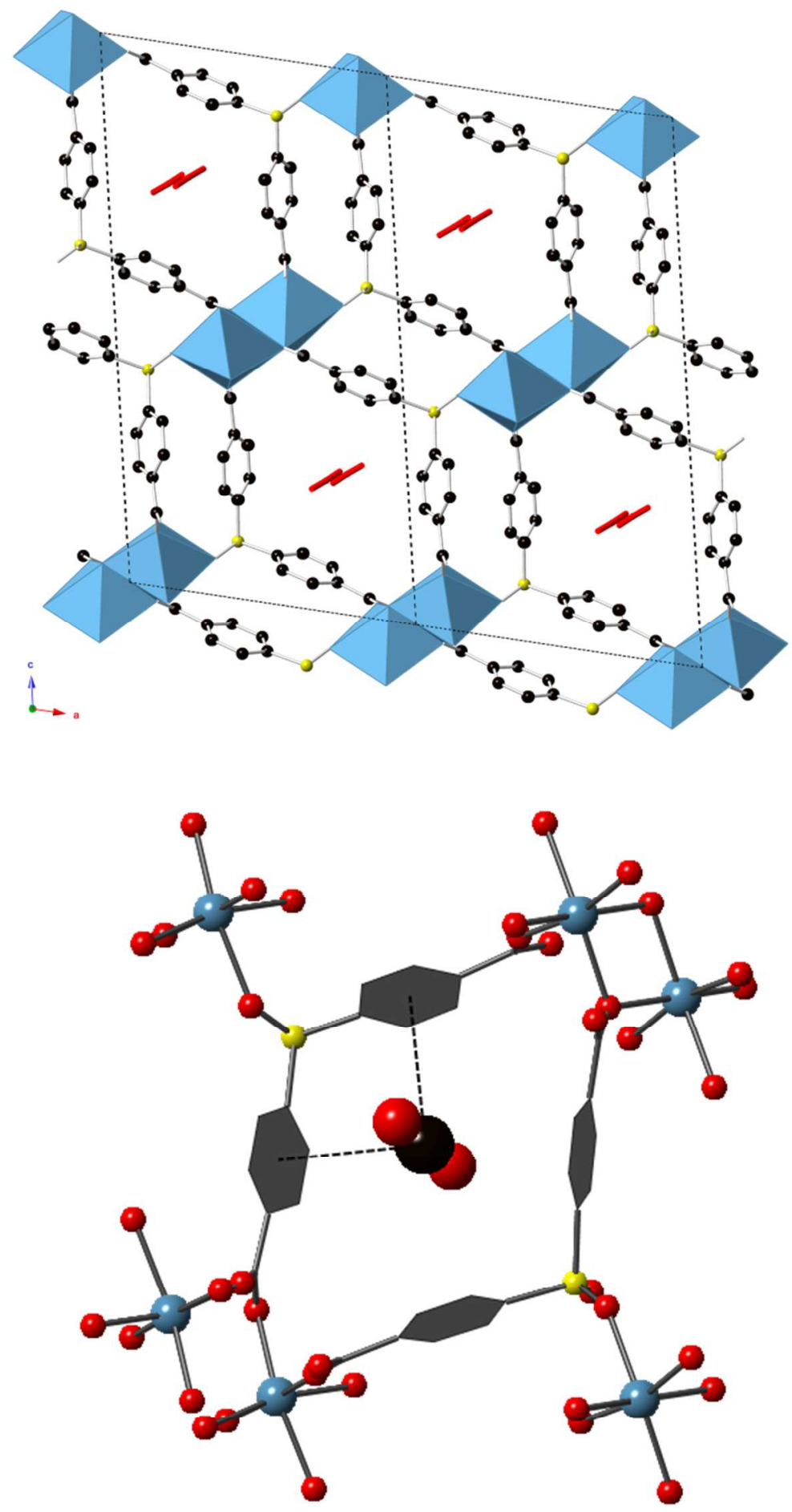

Figure S1. (top) Structure of SBMOF-1 at 1 atm of $\mathrm{CO}_{2}$. The calcium polyhedra are shown in blue, sulfur in yellow, carbon in black and $\mathrm{CO}_{2}$ in red. The $\mathrm{CO}_{2}$ molecules are coordinated to the two benzene rings. (bottom) Ball and stick model of the SBMOF-1 pore showing only one of the partially occupied $\mathrm{CO}_{2}$ sites coordinating to the benzene rings. 


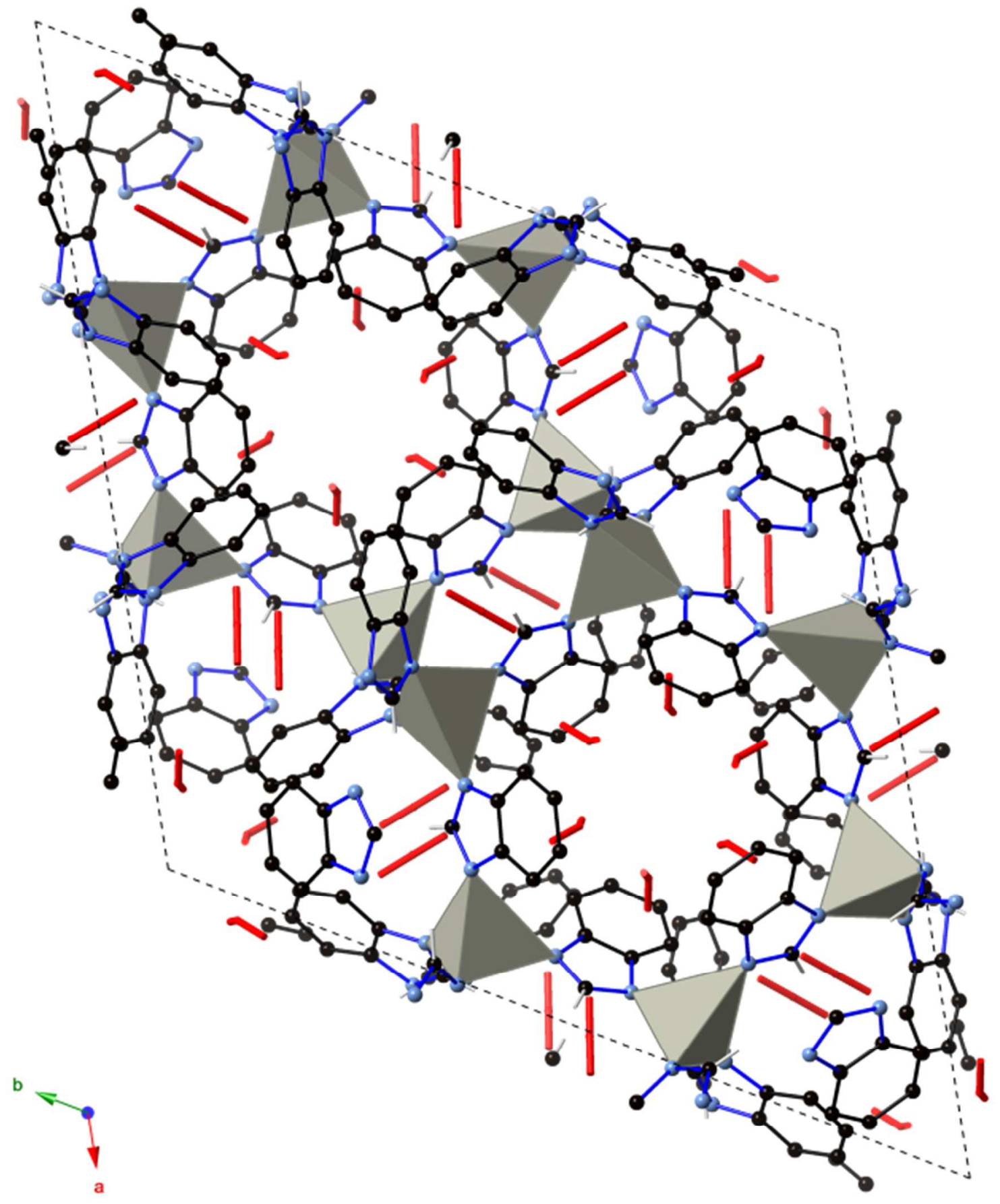

Figure S2. Structure of ZIF-7 (gate-open) at 1 atm of $\mathrm{CO}_{2}$. Zinc tetrahedra are shown in grey, nitrogen in blue, carbon in black and $\mathrm{CO}_{2}$ in red. The $\mathrm{CO}_{2}$ molecules are coordinated to the benzimidazolate rings. 


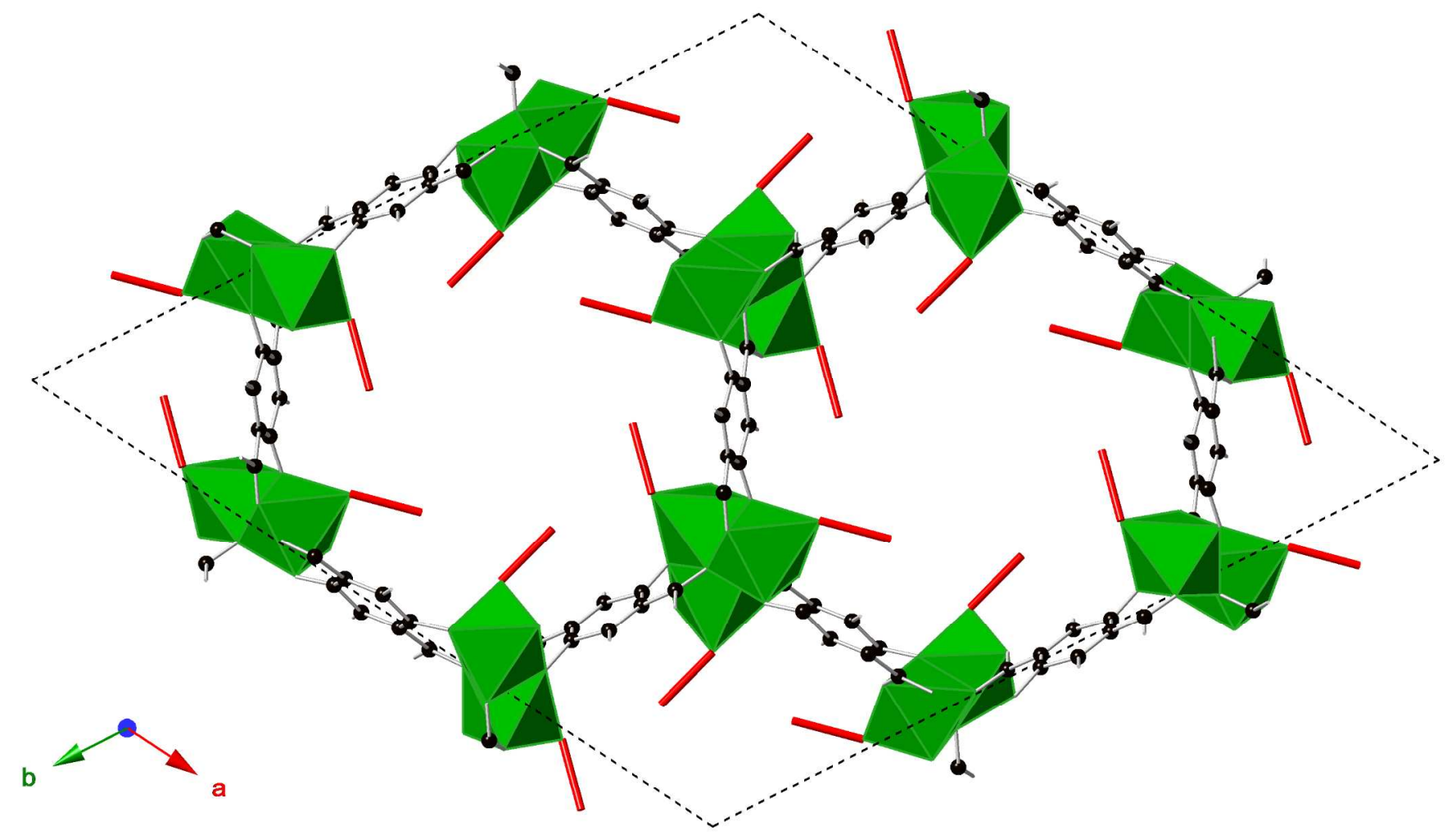

Figure S3. Structure of Ni-MOF-74 at 1 atm $\mathrm{CO}_{2}$. Nickel octahedra are green, carbon atoms are black, and $\mathrm{CO}_{2}$ is red. The $\mathrm{CO}_{2}$ molecules are coordinated to the $\mathrm{Ni}$ atoms and the oxygen atoms of $\mathrm{CO}_{2}$ complete the Ni octahedra. 


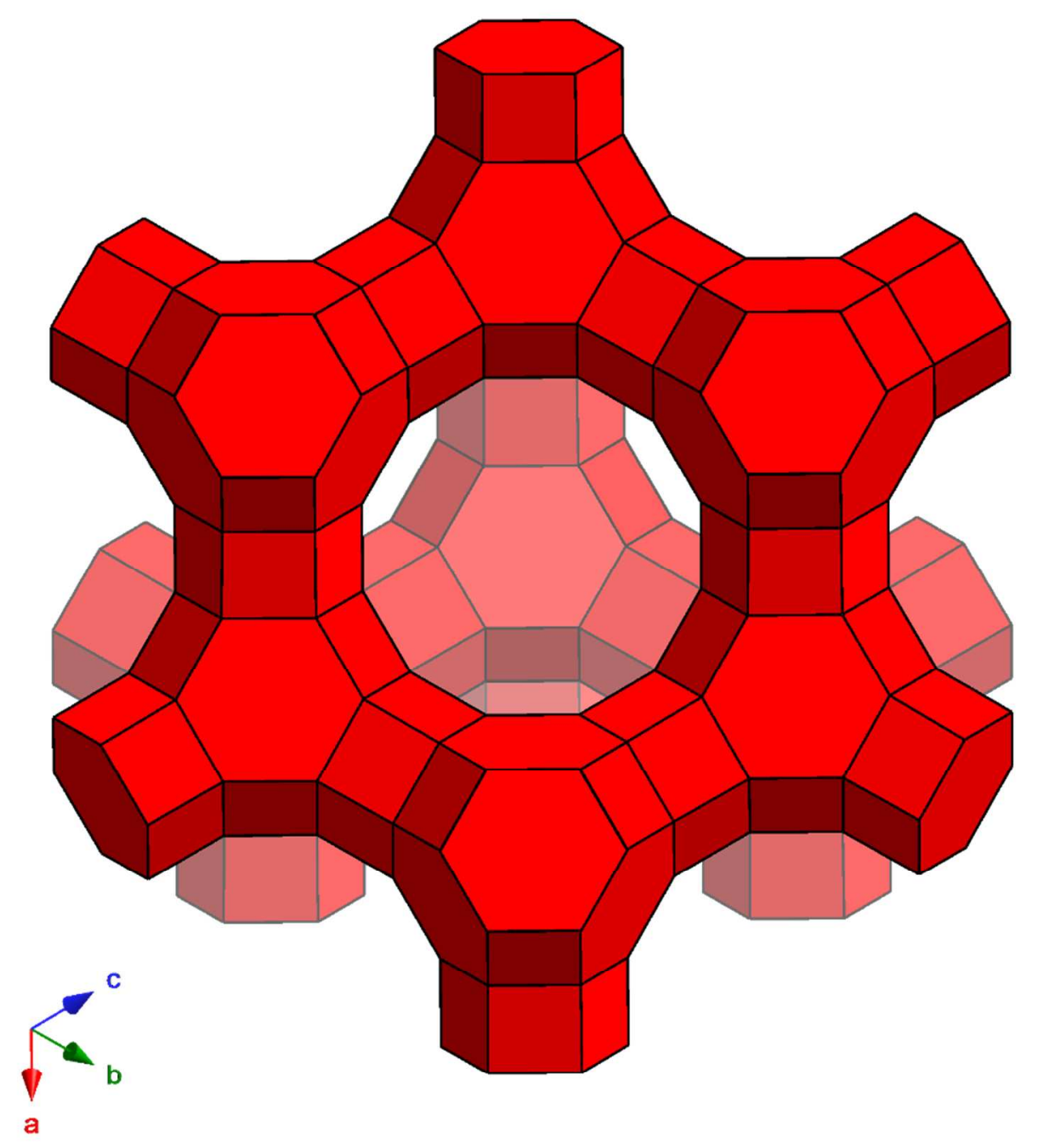

Figure S4. Framework structure of dehydrated zeolite NaX. The aluminosilicate network (red) is shown as polyhedral representation of the interconnected cages with the oxygen atoms and extra framework cations omitted for clarity. 

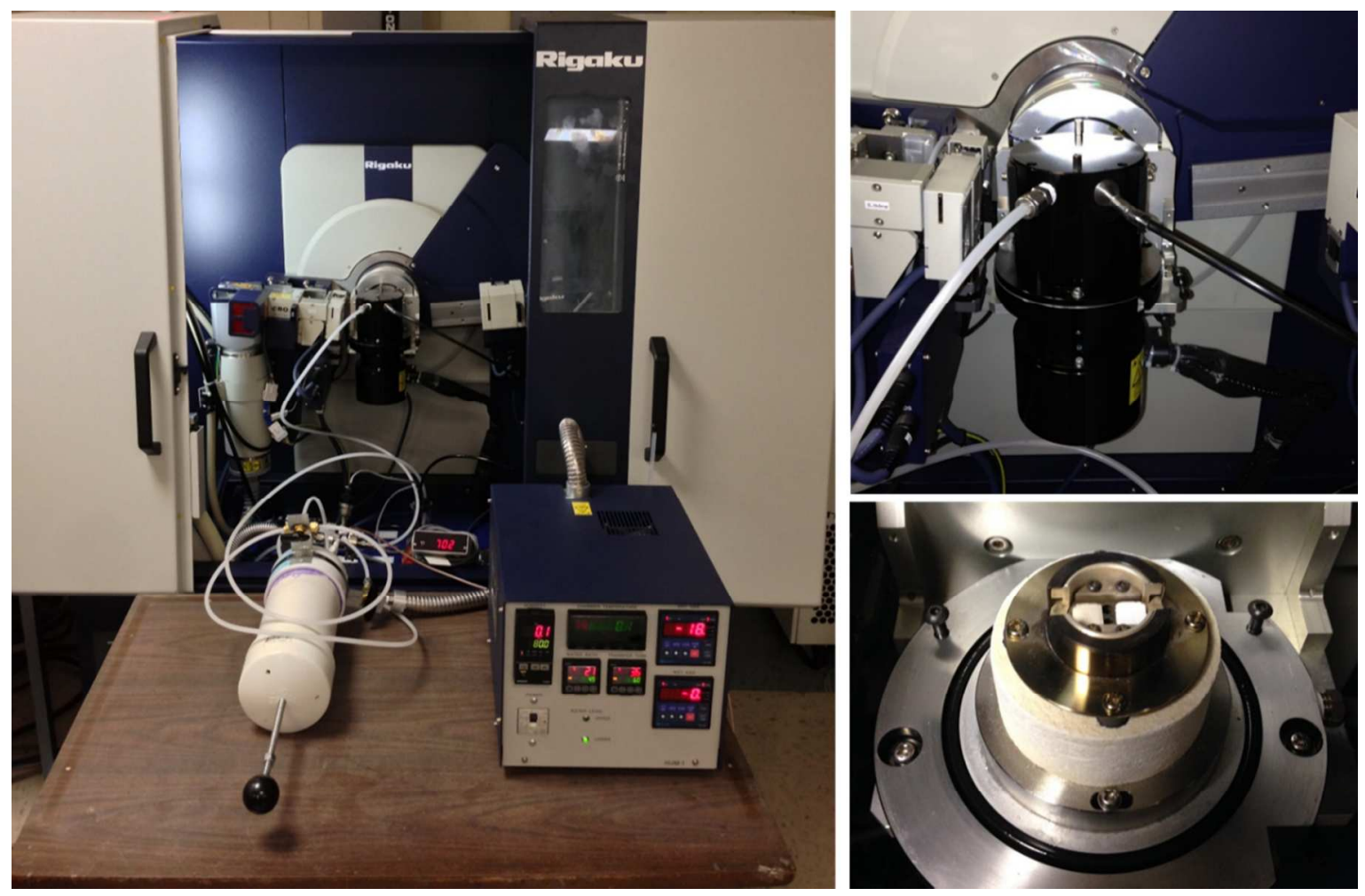

Figure S5. (Left) Photo of complete XRD-DSC system. (Top right) XRD-DSC stage with sealing cap. (Bottom right) XRD-DSC attachment without cap showing the $\mathrm{Al}_{2} \mathrm{O}_{3}$ standard and sample powder on aluminum pans. 


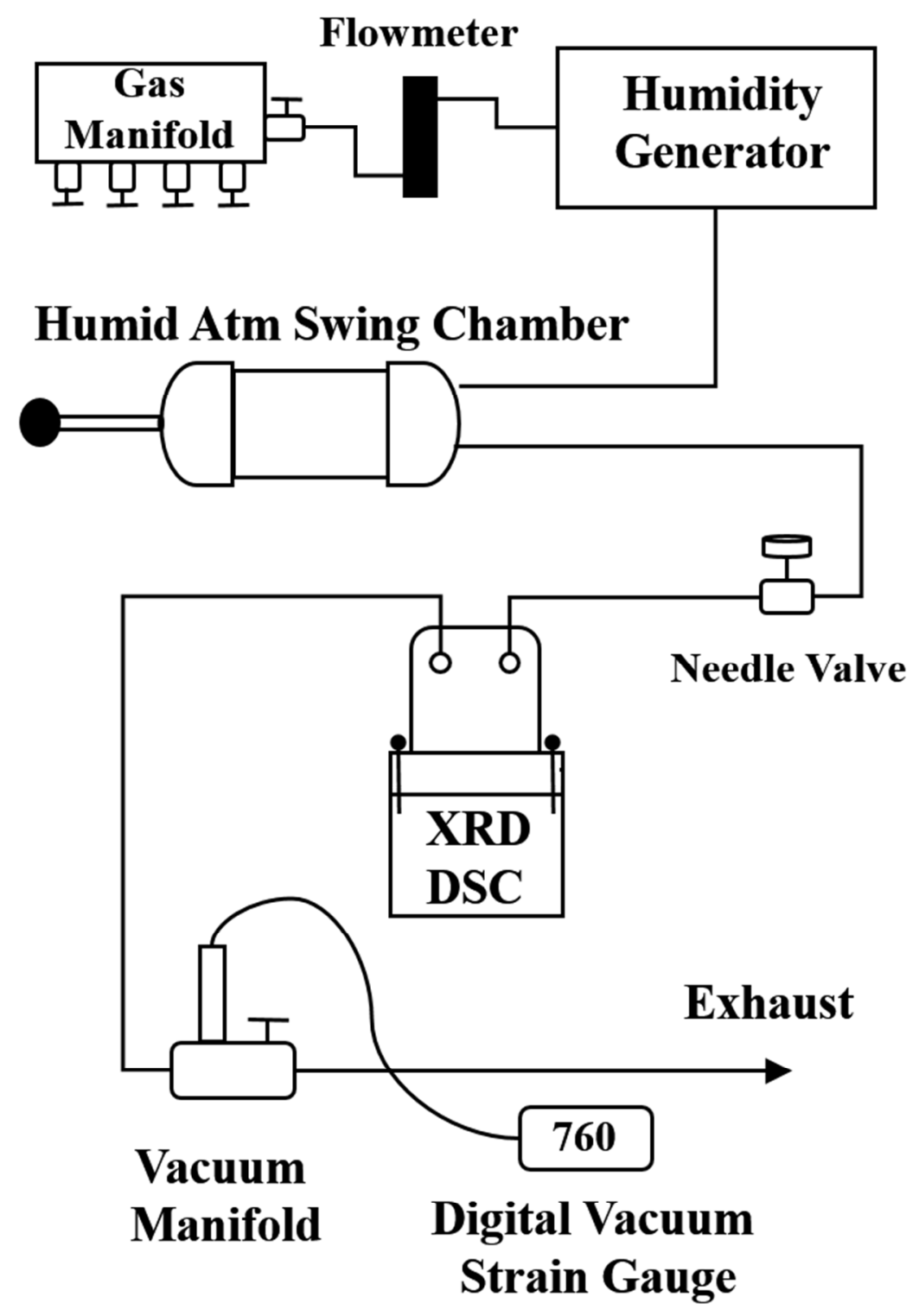

Figure S6. Schematic of entire XRD - DSC system. 


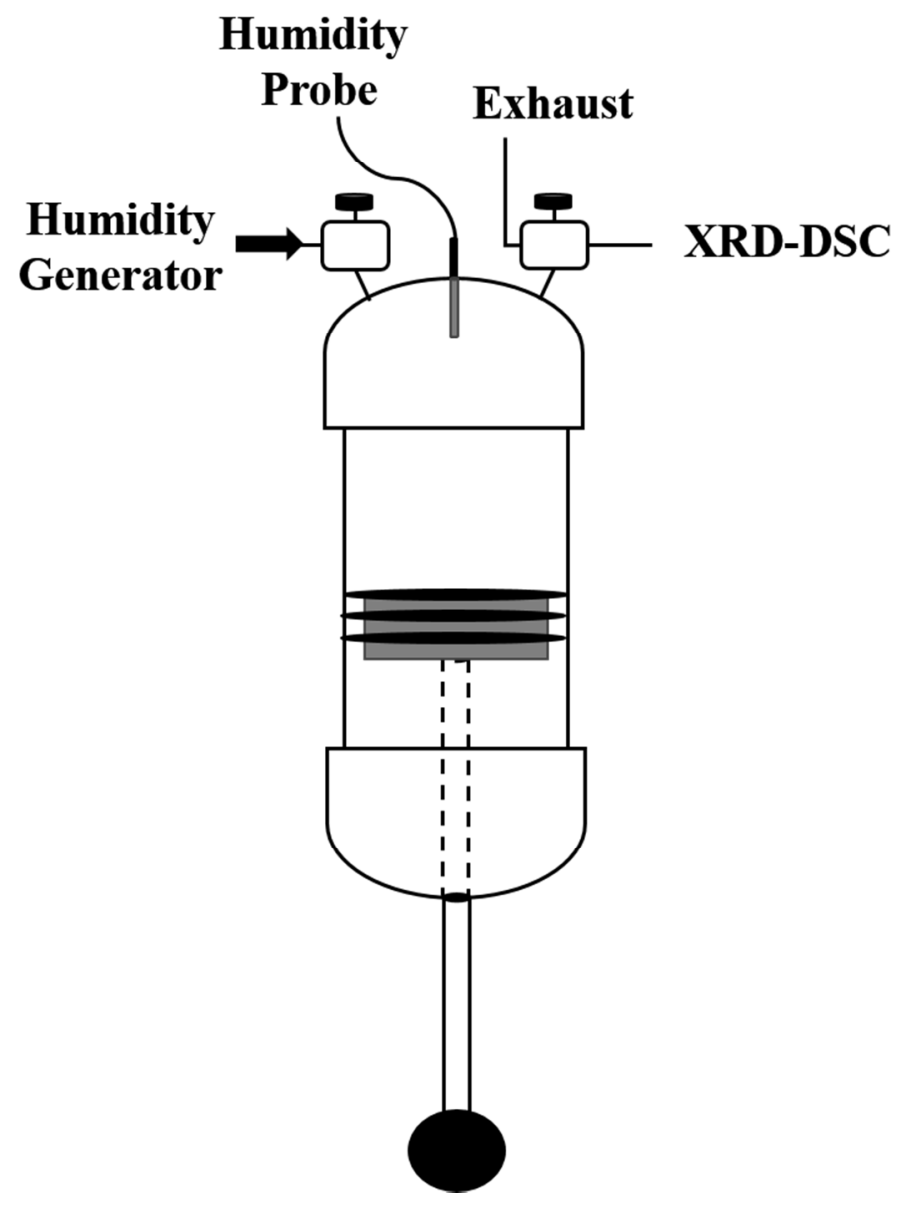

Figure S7. Schematic of humid atmosphere swing chamber (HASC). The atmosphere is input from the humidity generator which controls the mixing of wet and dry mass flow controllers based on feedback from a humidity probe sealed in the top of the chamber. The base of the chamber is designed like a syringe with a triple O-ring seal so the volume of the chamber can be changed without atmospheric contamination. 


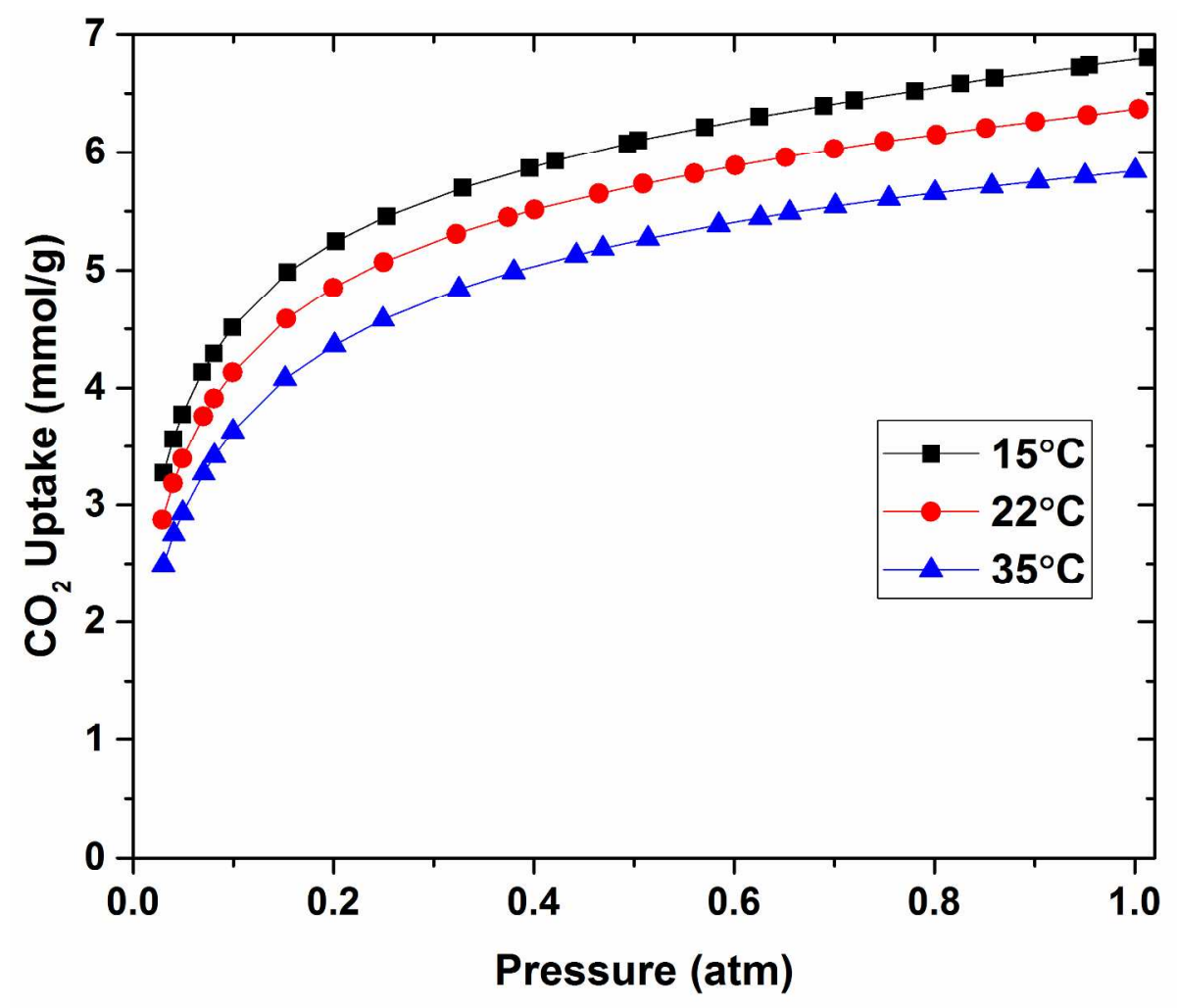

Figure S8. $\mathrm{NaX} \mathrm{CO}_{2}$ adsorption isotherms.

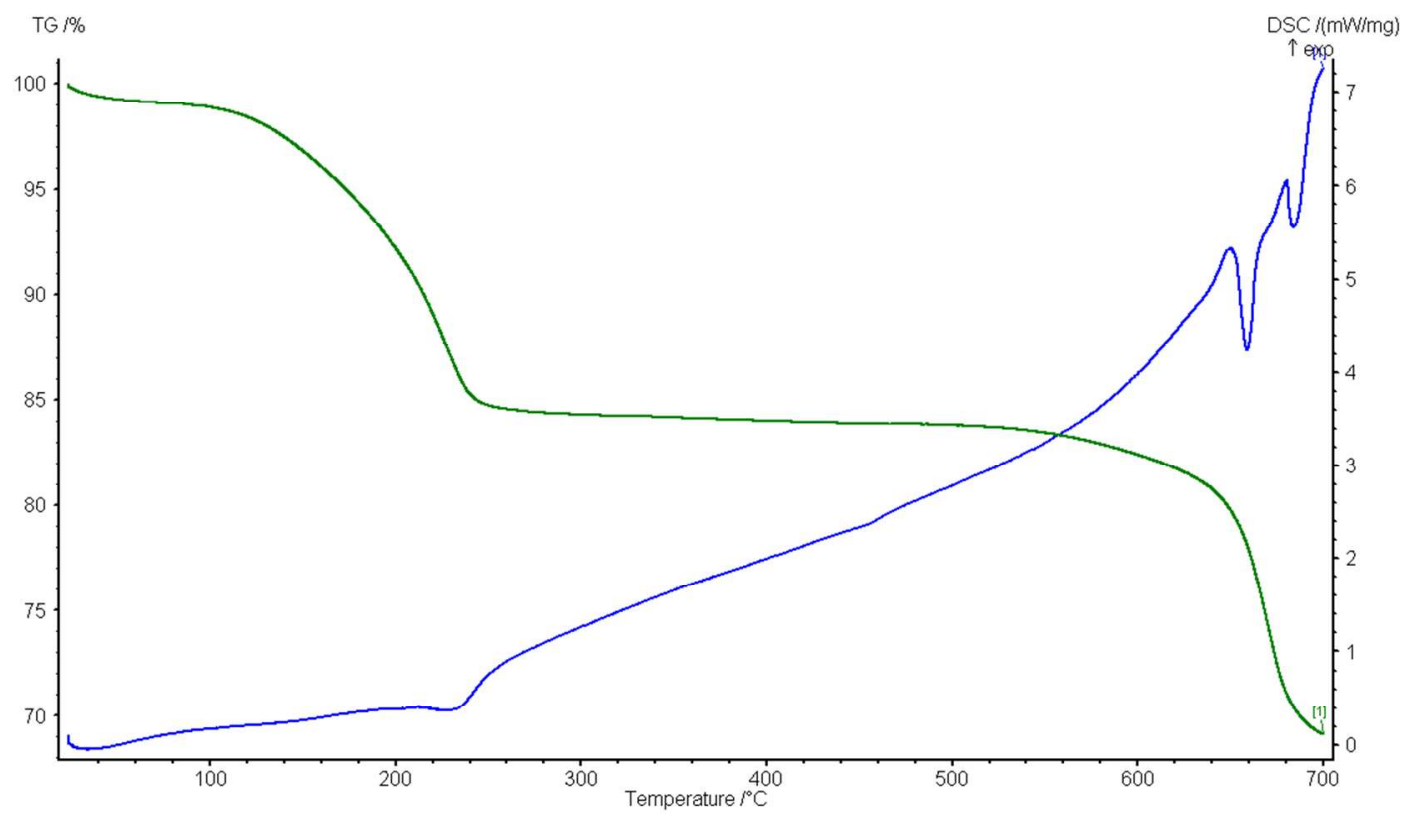

Figure S9. TGA plot of the ZIF-7 material 


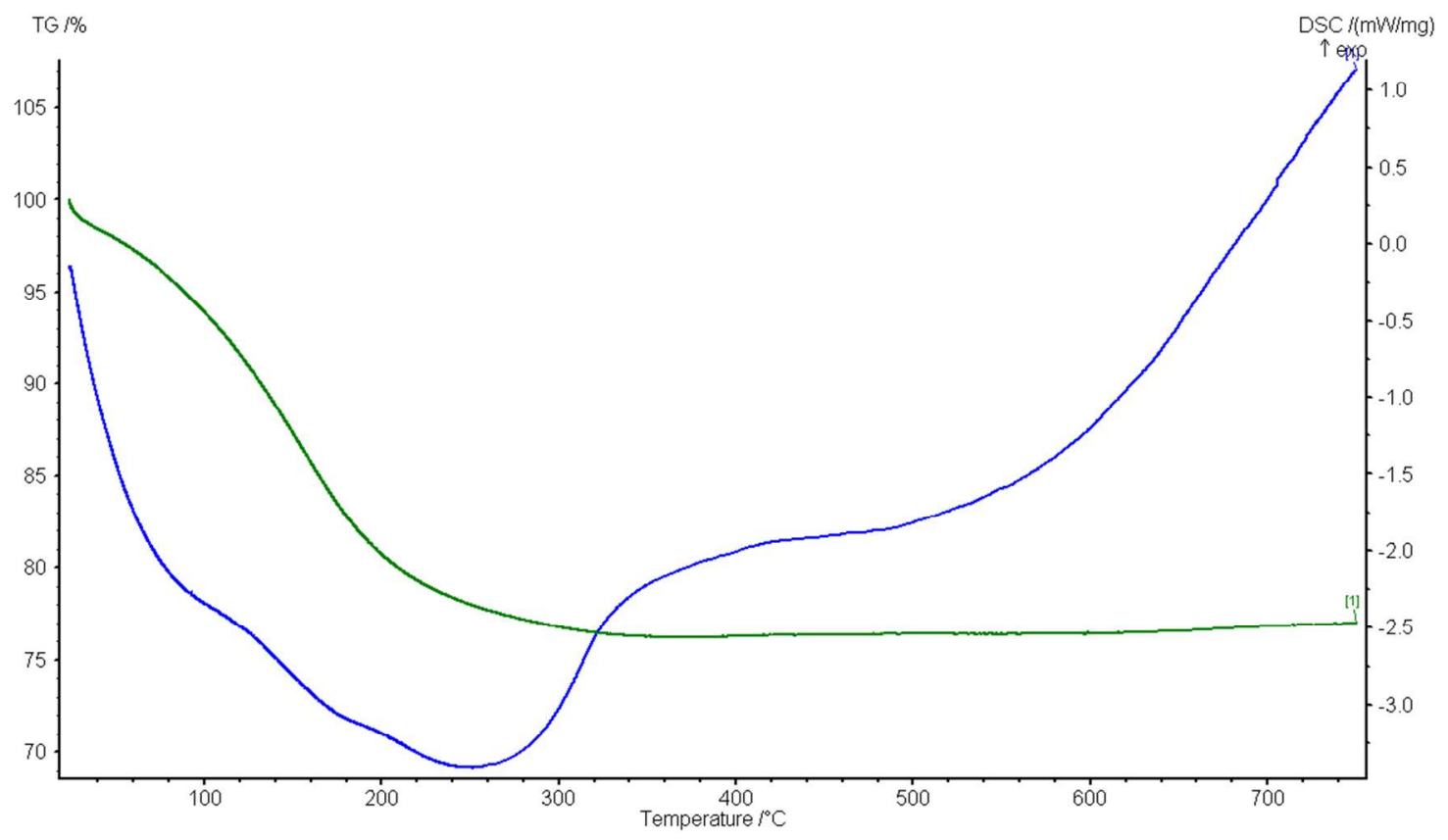

Figure S10. TGA plot of the $\mathrm{NaX}$ material

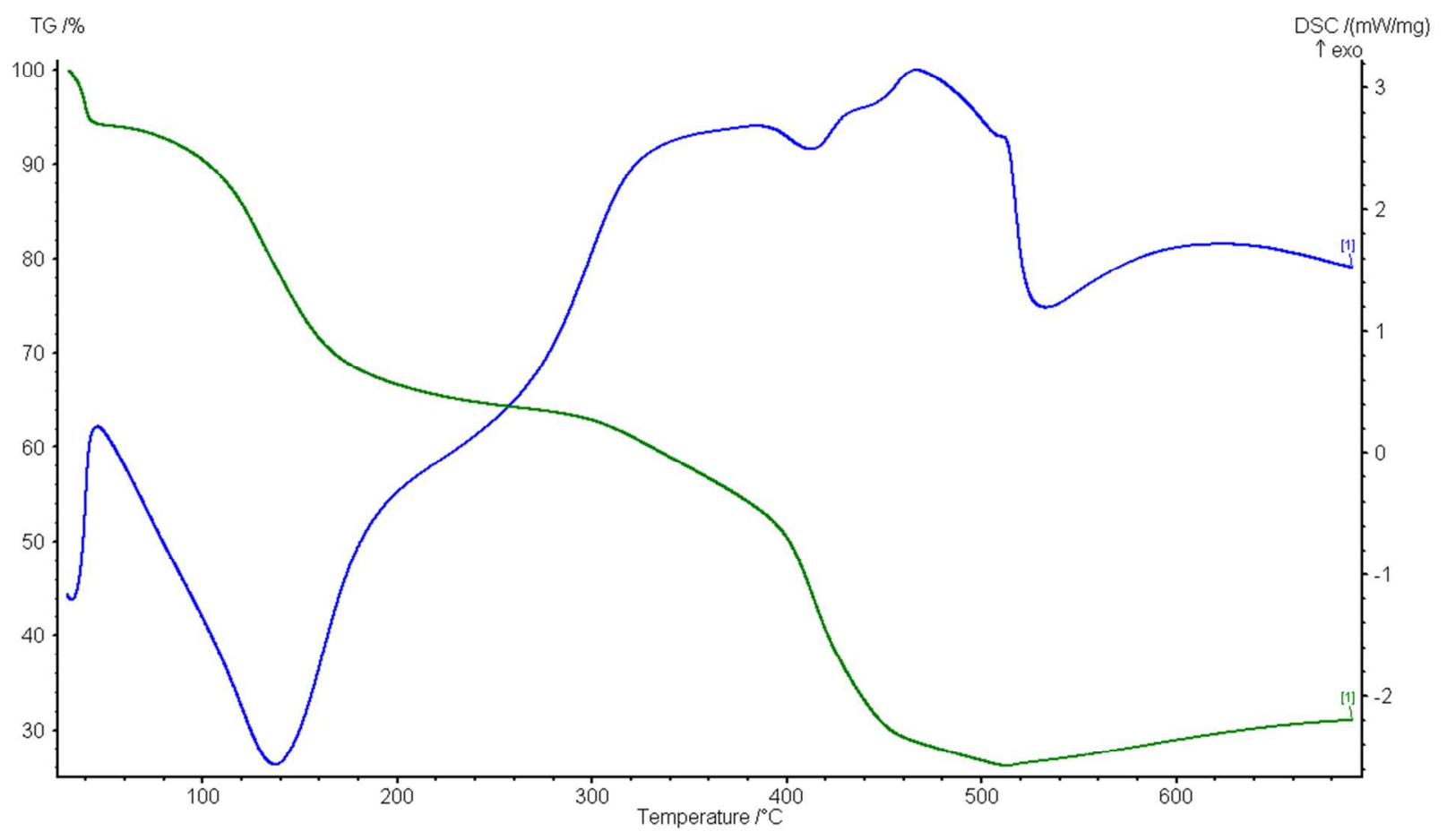

Figure S11. TGA plot of the Ni MOF-74 materia 

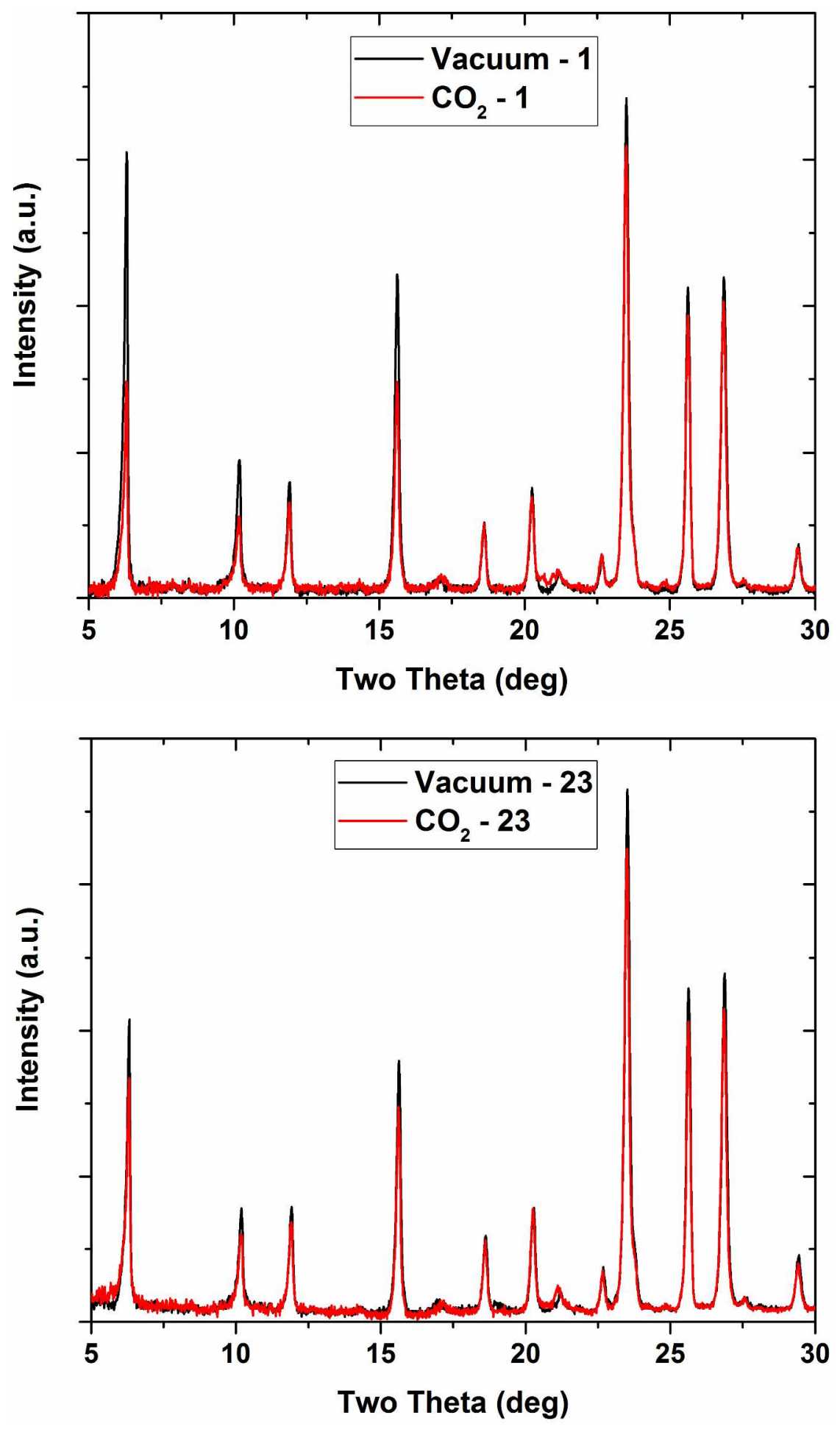

Figure S12. Comparison of the $\mathrm{NaX} \mathrm{XRD}$ patterns collected under vacuum and $25 \% \mathrm{RH} \mathrm{CO}$ during cycle 1 (top) and cycle 23 (bottom). 

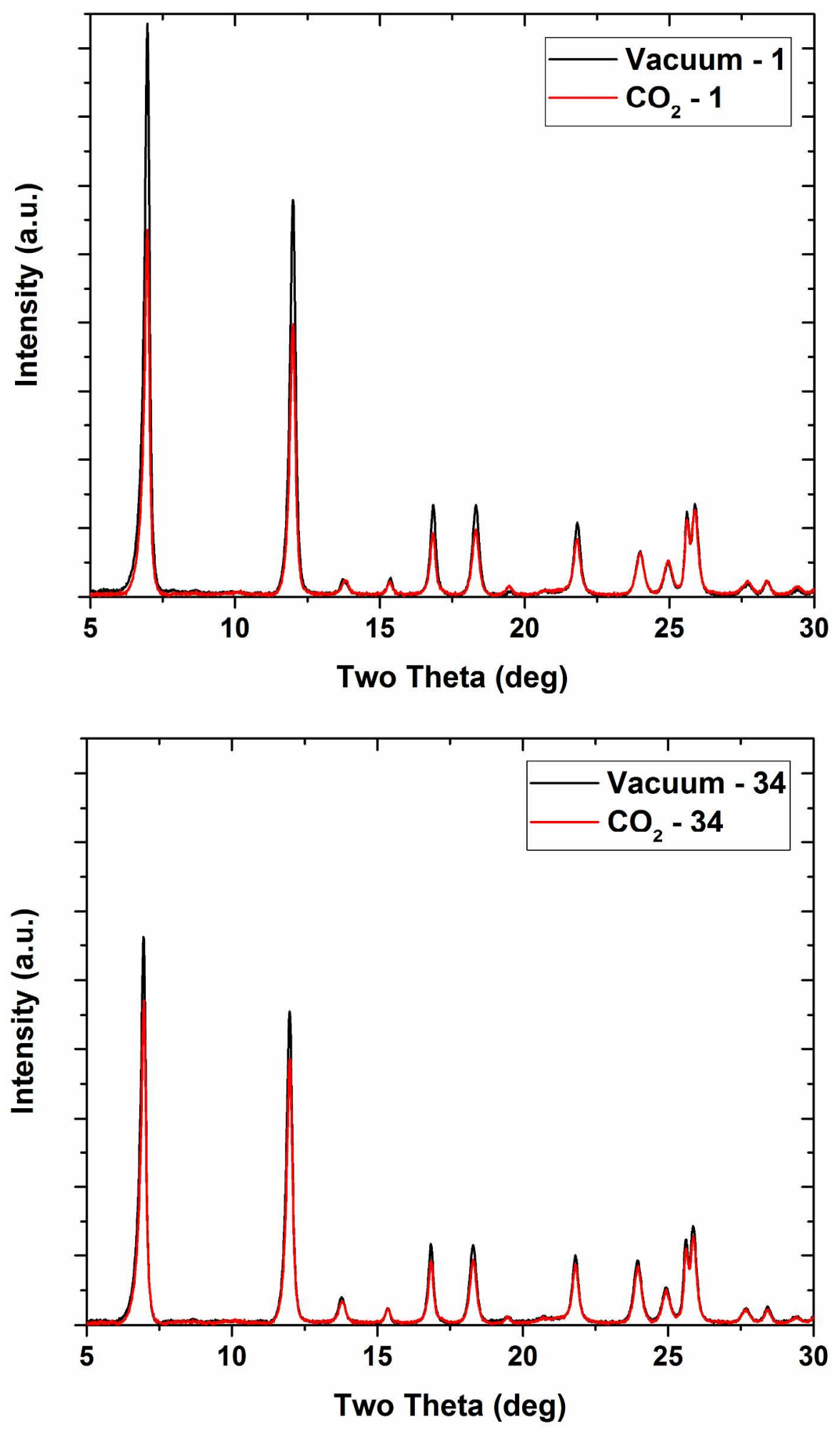

Figure S13. Comparison of the Ni-MOF-74 XRD patterns collected under vacuum and 25\% RH $\mathrm{CO}_{2}$ during cycle 1 (top) and cycle 34 (bottom). 


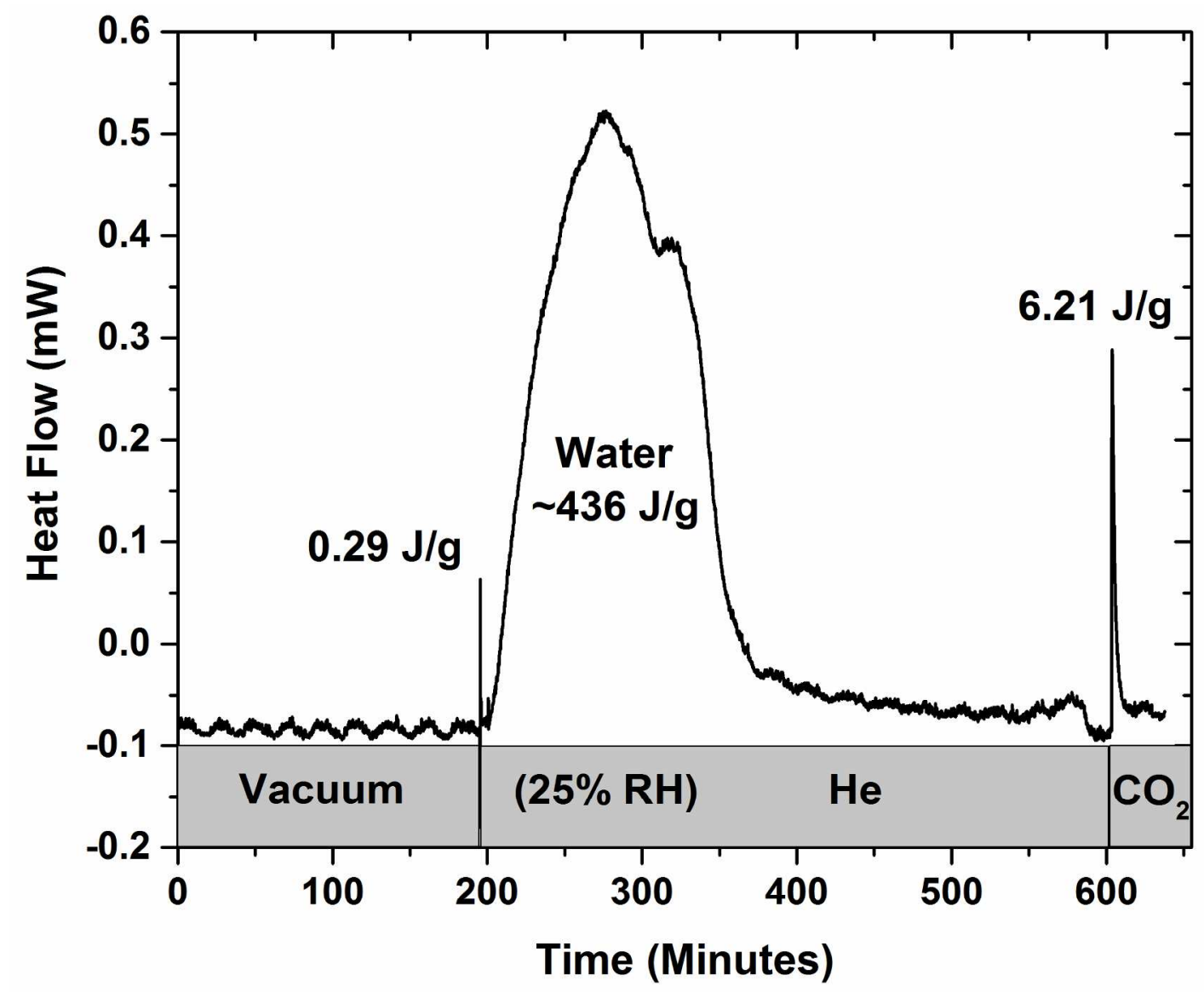

Figure S14. DSC signal during Ni-MOF-74 humid atmosphere $(25 \% \mathrm{RH})$ swing. Activated NiMOF-74 under vacuum is first exposed to helium gas at $25 \% \mathrm{RH}$ at a constant flow of 150 $\mathrm{mL} / \mathrm{min}$, then to an atmosphere of $\mathrm{CO}_{2}$ at $25 \% \mathrm{RH}$. After exposure to the $25 \% \mathrm{RH}$ helium stream, a large exotherm is observed due to the slow adsorption of water. The aberration in the signal between $\sim 560$ and 600 minutes is due to an electronic noise contribution from collecting the XRD pattern. 

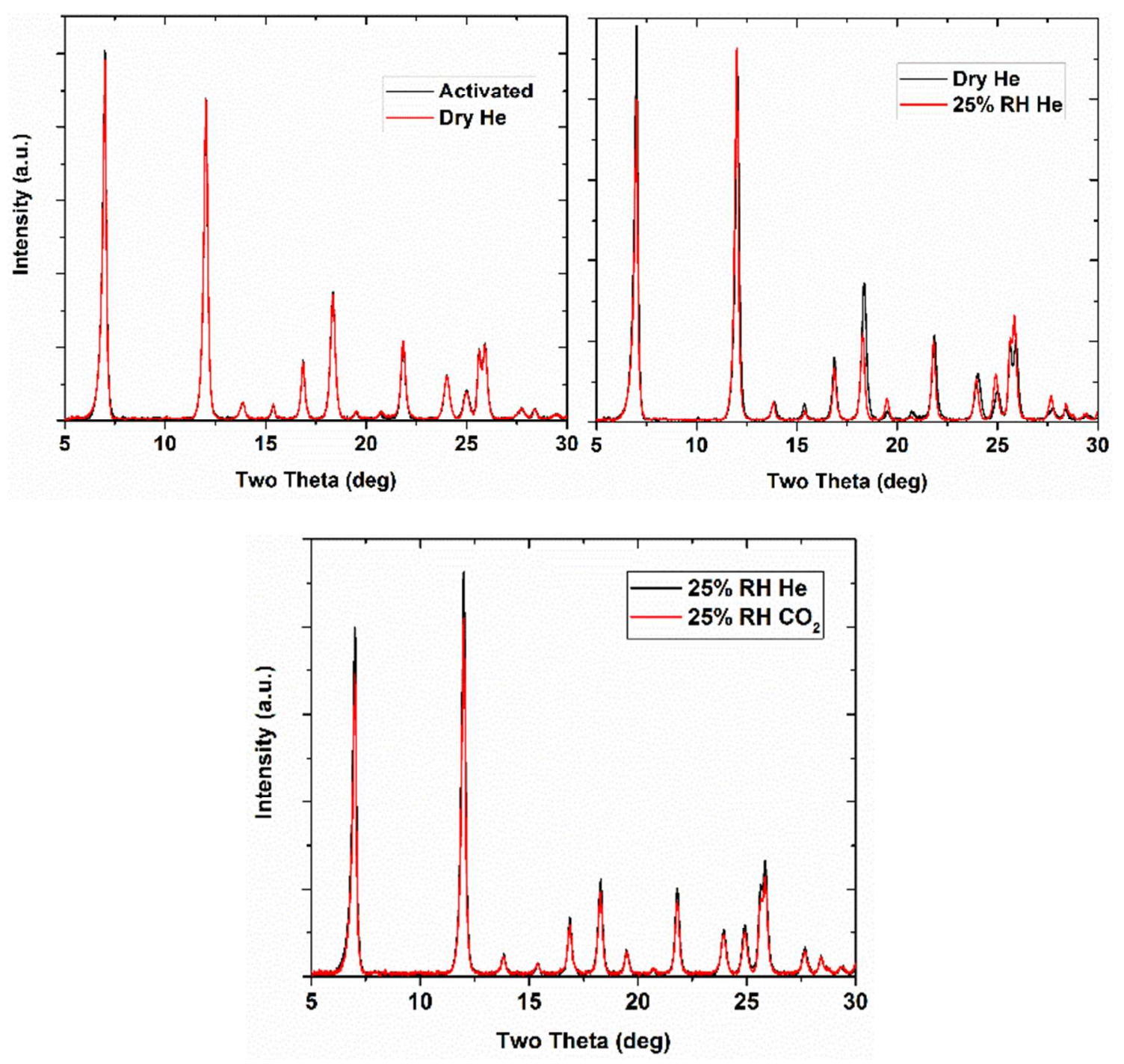

Figure S15. XRD patterns of Ni-MOF-74 under different gas loading conditions. (top left) Activated Ni-MOF-74 under vacuum vs dry helium. (top right) Ni-MOF-74 under dry helium vs after exposure to $25 \% \mathrm{RH}$ helium. (bottom) Ni-MOF-74 under $25 \% \mathrm{RH}$ helium vs $25 \% \mathrm{RH} \mathrm{CO}_{2}$. 


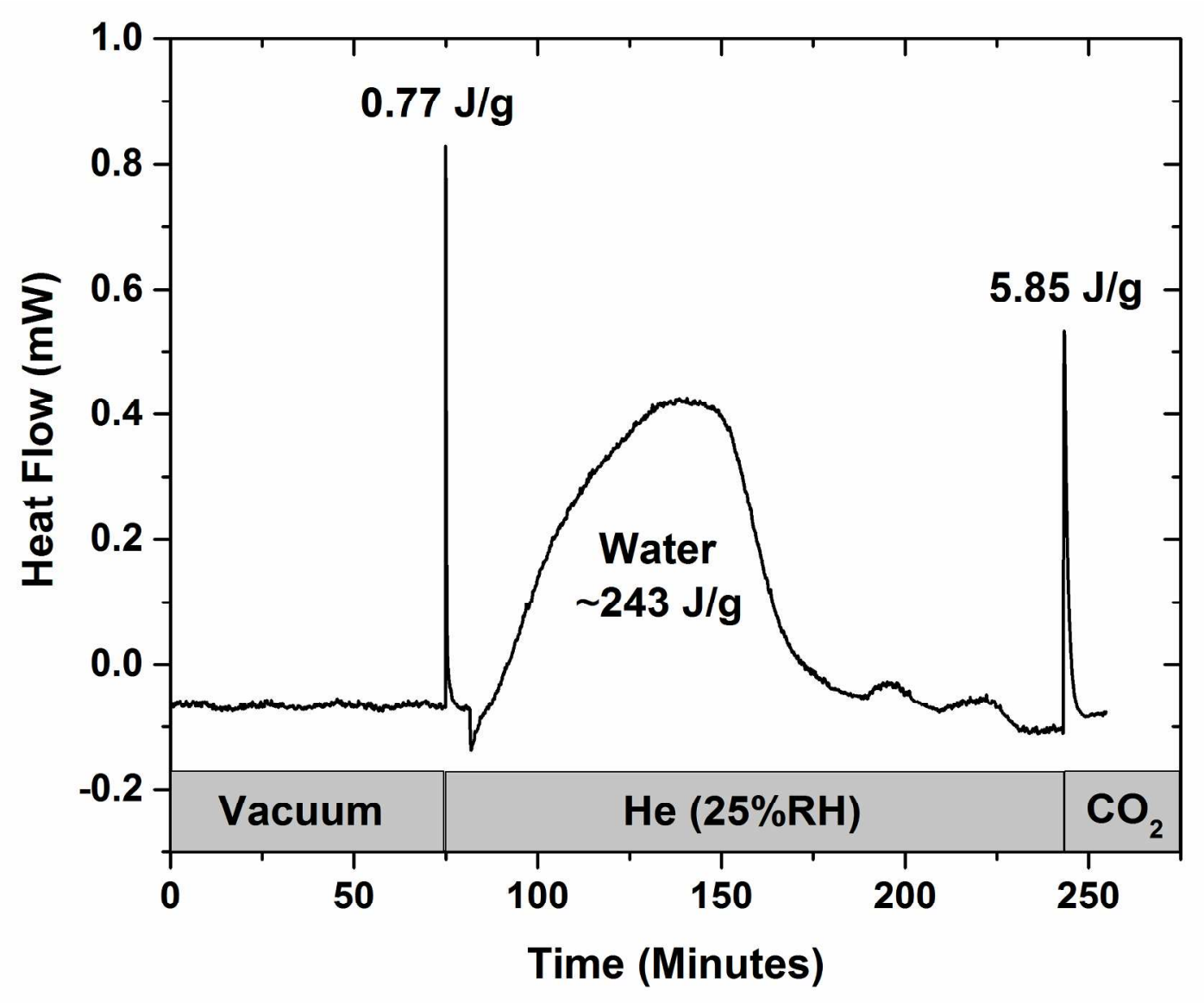

Figure S16. DSC signal during $\mathrm{NaX}$ humid atmosphere $(25 \% \mathrm{RH})$ swing. Activated $\mathrm{NaX}$ under vacuum is first exposed to helium gas at $25 \% \mathrm{RH}$ at a constant flow of $150 \mathrm{~mL} / \mathrm{min}$, then to an atmosphere of $\mathrm{CO}_{2}$ at $25 \% \mathrm{RH}$. After exposure to the $25 \% \mathrm{RH}$ helium stream, a large exotherm is observed due to the slow adsorption of water. 

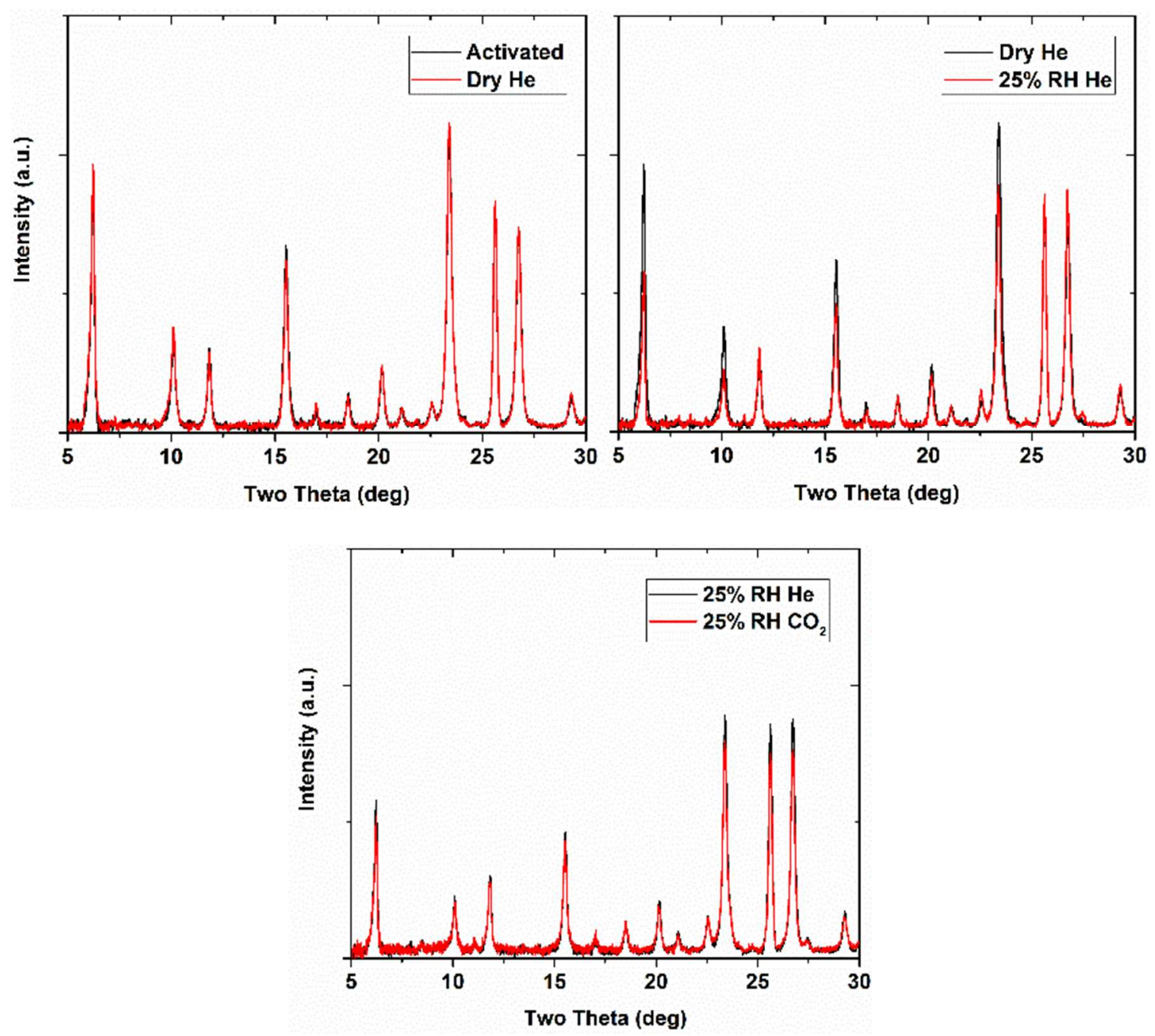

Figure S17. XRD patterns of $\mathrm{NaX}$ under different gas loading conditions. (top left) Activated $\mathrm{NaX}$ under vacuum vs dry helium. (top right) $\mathrm{NaX}$ under dry helium vs after exposure to $25 \%$ RH helium. (bottom) $\mathrm{NaX}$ under $25 \% \mathrm{RH}$ helium vs $25 \% \mathrm{RH} \mathrm{CO}_{2}$. 


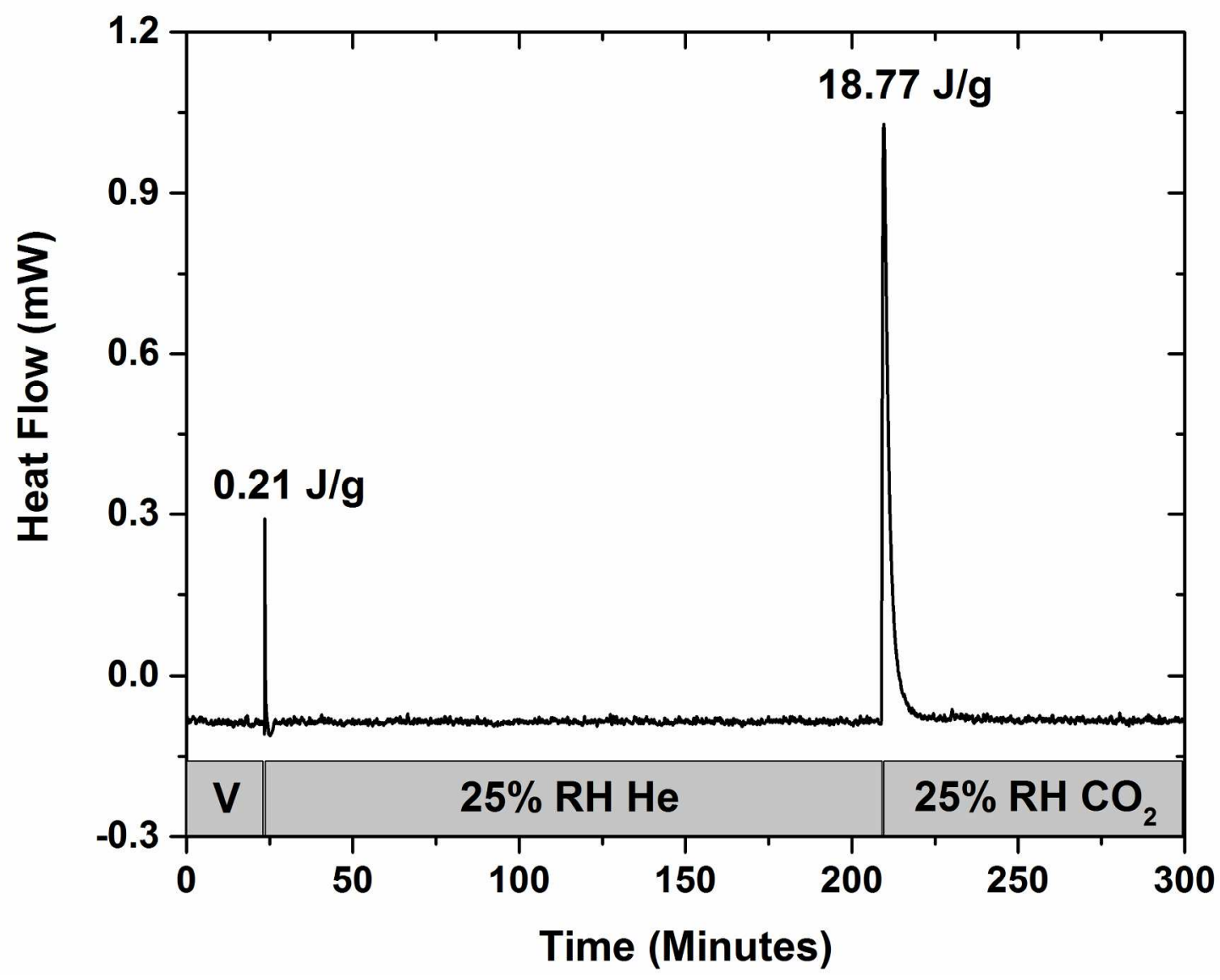

Figure S18. DSC signal during SBMOF-1 humid atmosphere (25\% RH) swing. Activated SBMOF-1 under vacuum is first exposed to helium gas at $25 \% \mathrm{RH}$ at a constant flow of 150 $\mathrm{mL} / \mathrm{min}$, then to an atmosphere of $\mathrm{CO}_{2}$ at $25 \% \mathrm{RH}$. 

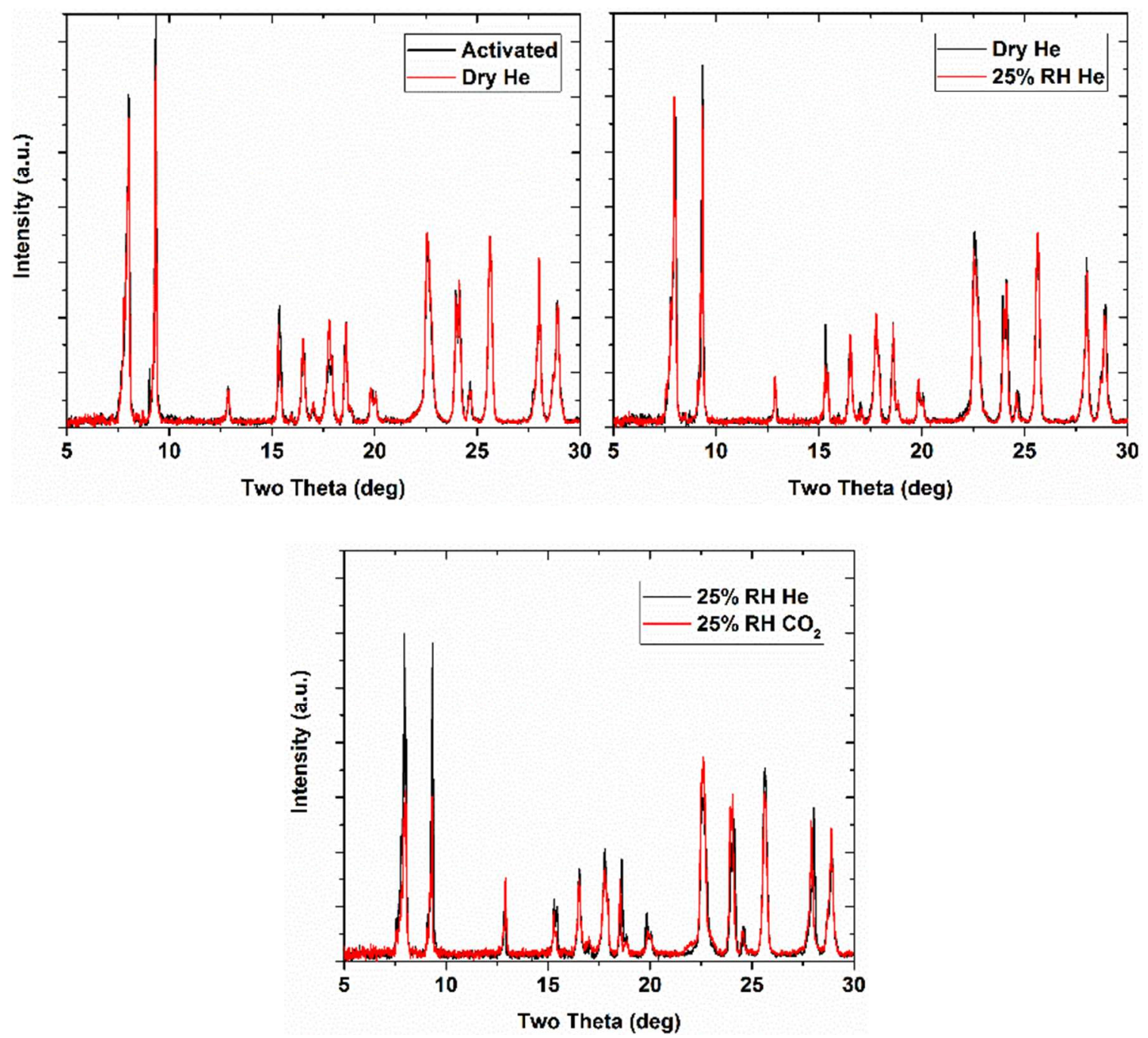

Figure S19. XRD patterns of SBMOF-1 under different gas loading conditions. (left) Activated SBMOF-1 under vacuum vs dry helium. (middle) SBMOF-1 under dry helium vs after exposure to $25 \% \mathrm{RH}$ helium. (right) SBMOF-1 under $25 \% \mathrm{RH}$ helium vs $25 \% \mathrm{RH} \mathrm{CO}_{2}$. 


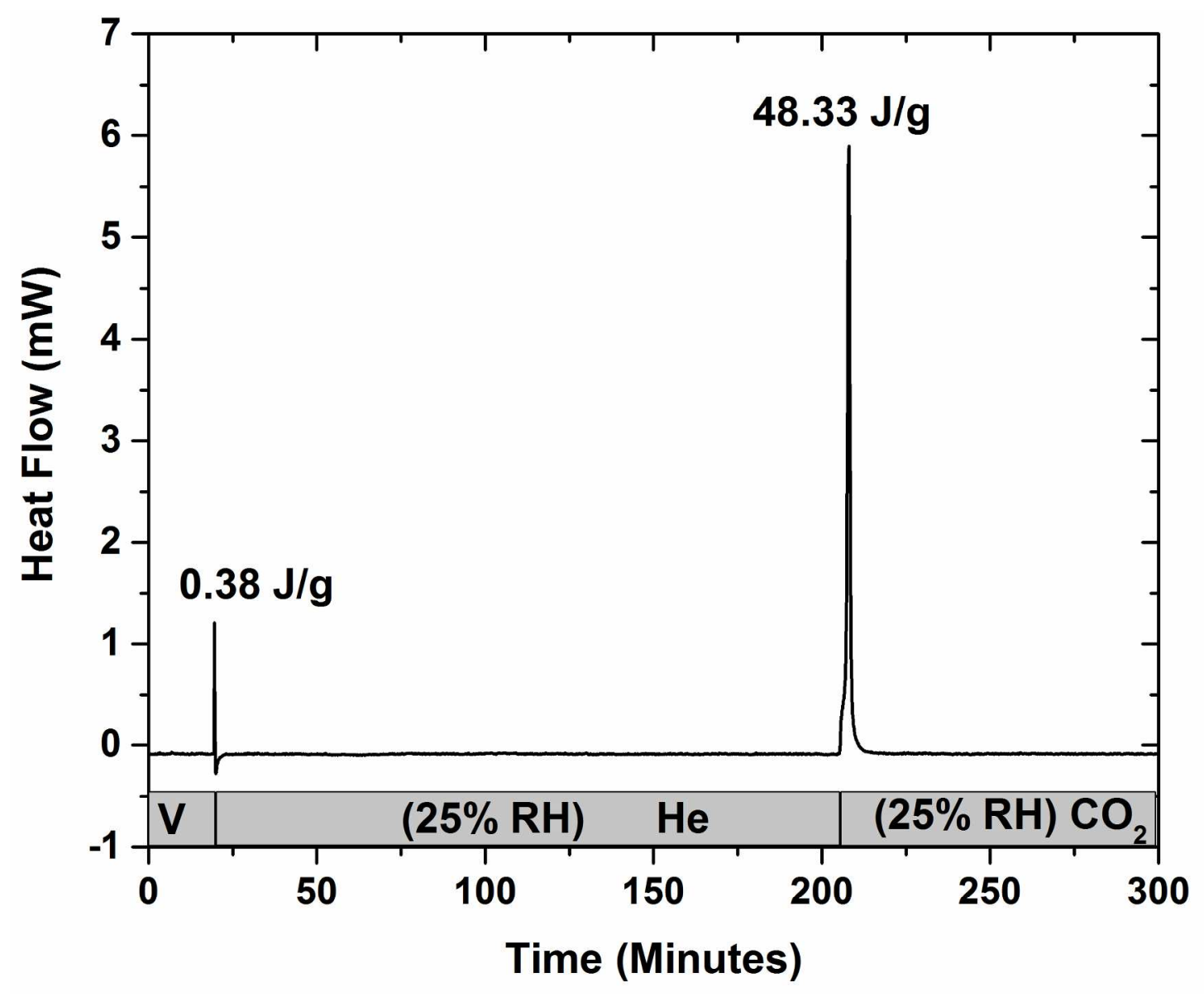

Figure S20. DSC signal during ZIF-7 humid atmosphere (25\% RH) swing. Activated ZIF-7 under vacuum (V) is first exposed to helium gas at $25 \% \mathrm{RH}$ at a constant flow of $150 \mathrm{~mL} / \mathrm{min}$, then to an atmosphere of $\mathrm{CO}_{2}$ at $25 \% \mathrm{RH}$. 

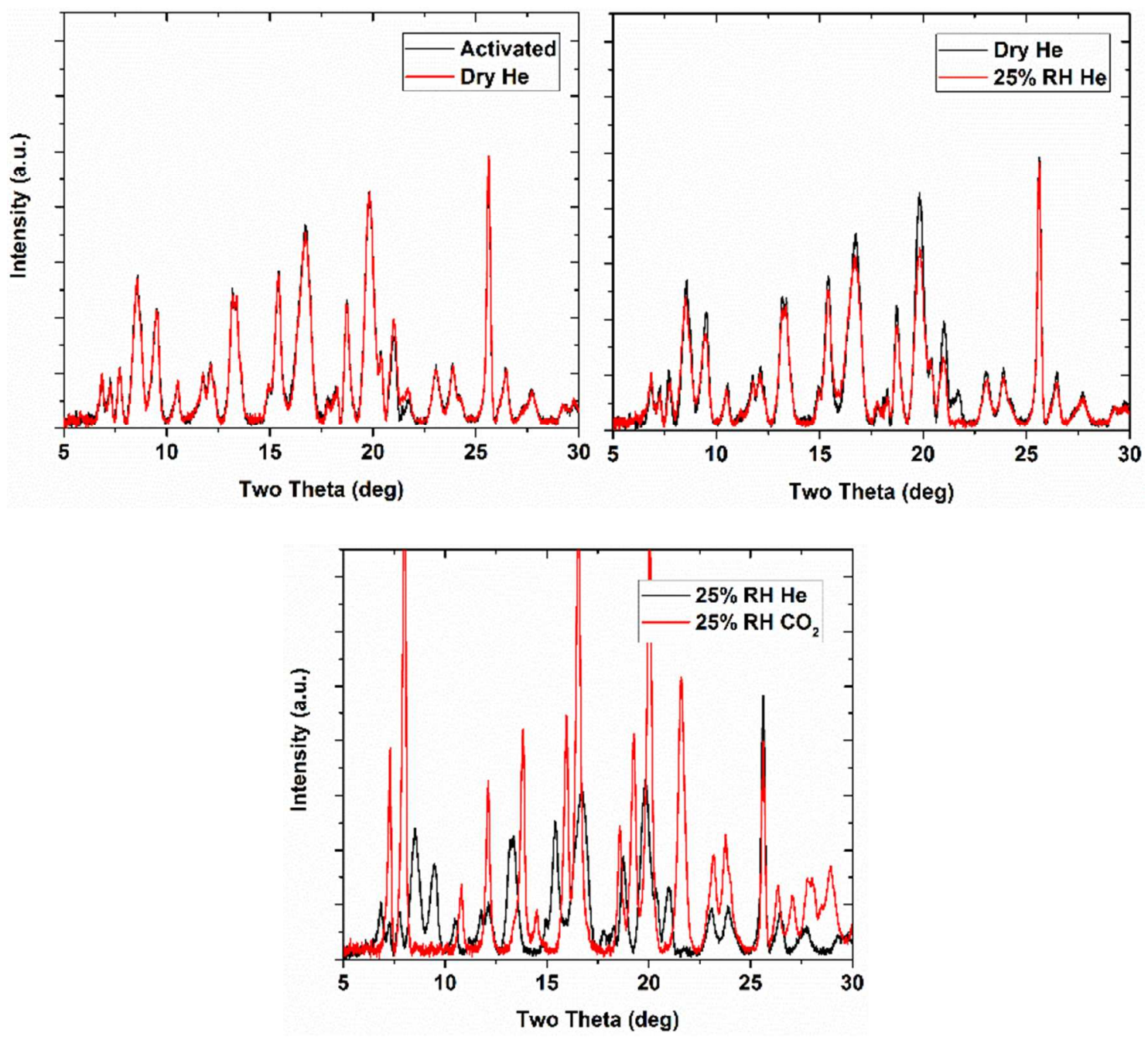

Figure S21. XRD patterns of ZIF-7 under different gas loading conditions. (top left) Activated ZIF-7 under vacuum vs dry helium. (top right) ZIF-7 under dry helium vs after exposure to $25 \%$ $\mathrm{RH}$ helium. (bottom) ZIF-7 under $25 \% \mathrm{RH}$ helium vs $25 \% \mathrm{RH} \mathrm{CO}$. 


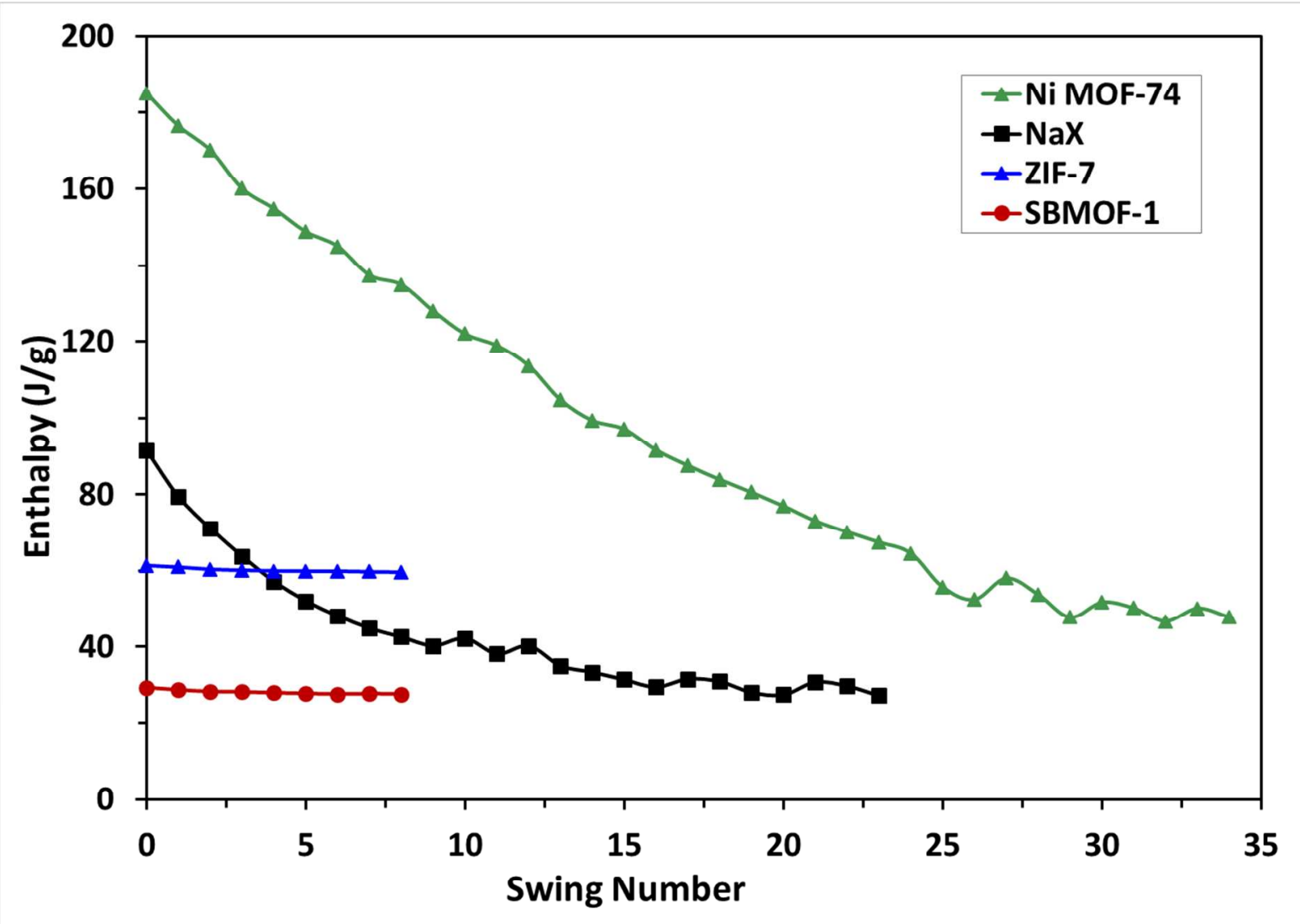

Figure S22. The measured enthalpy of successive vacuum-CO2 gas loading swings (1 atm) at $25 \%$ relative humidity and $22{ }^{\circ} \mathrm{C}$ for Ni-MOF74 (green), NaX (black), ZIF-7 (blue) and SBMOF-1 (red). 


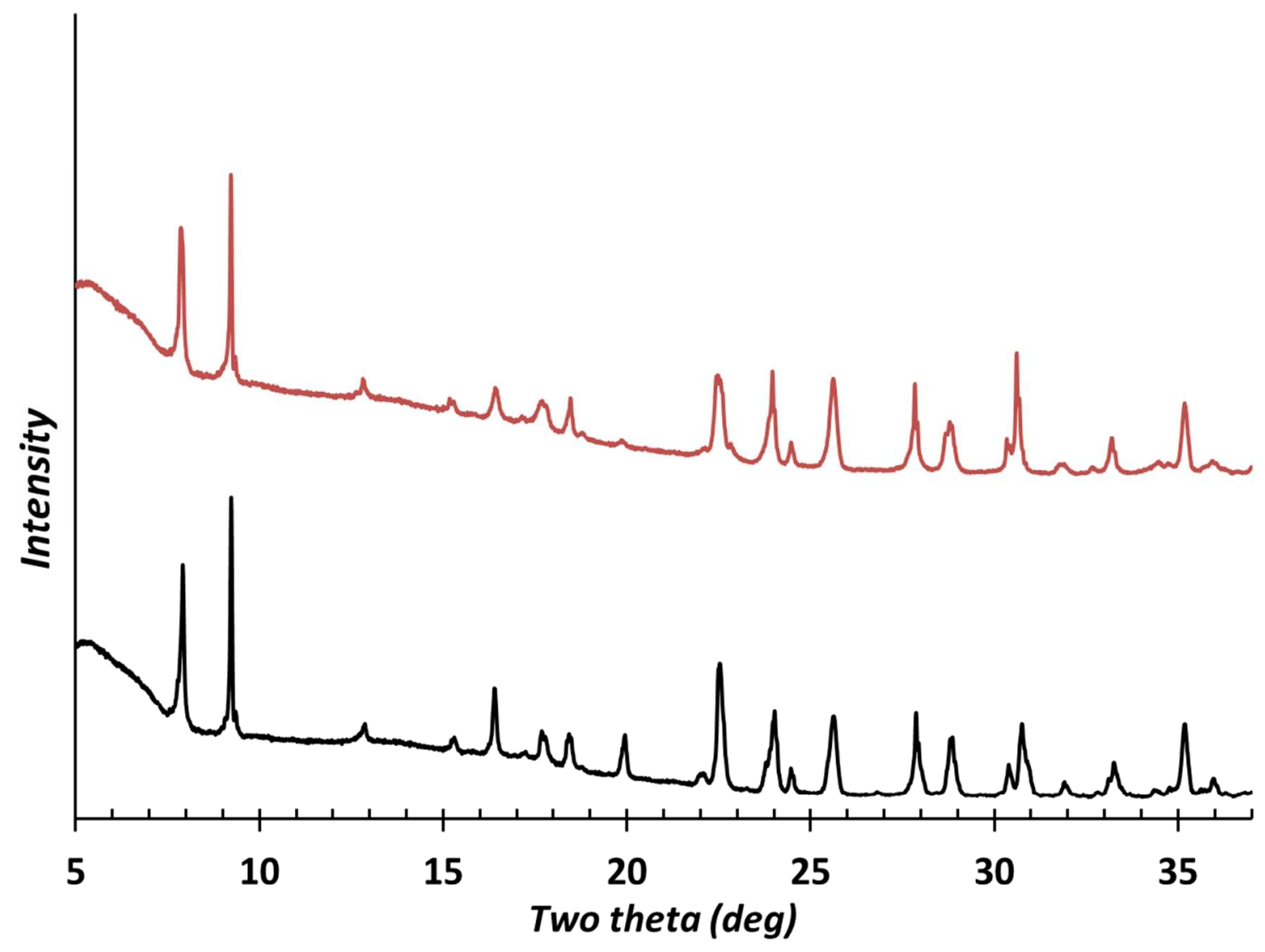

Figure S23. PXRD patterns of as-synthesized (black) and final (red) SBMOF-1 (after 12 swings of $\mathrm{CO}_{2}$ under $75 \% \mathrm{RH}$ ). 


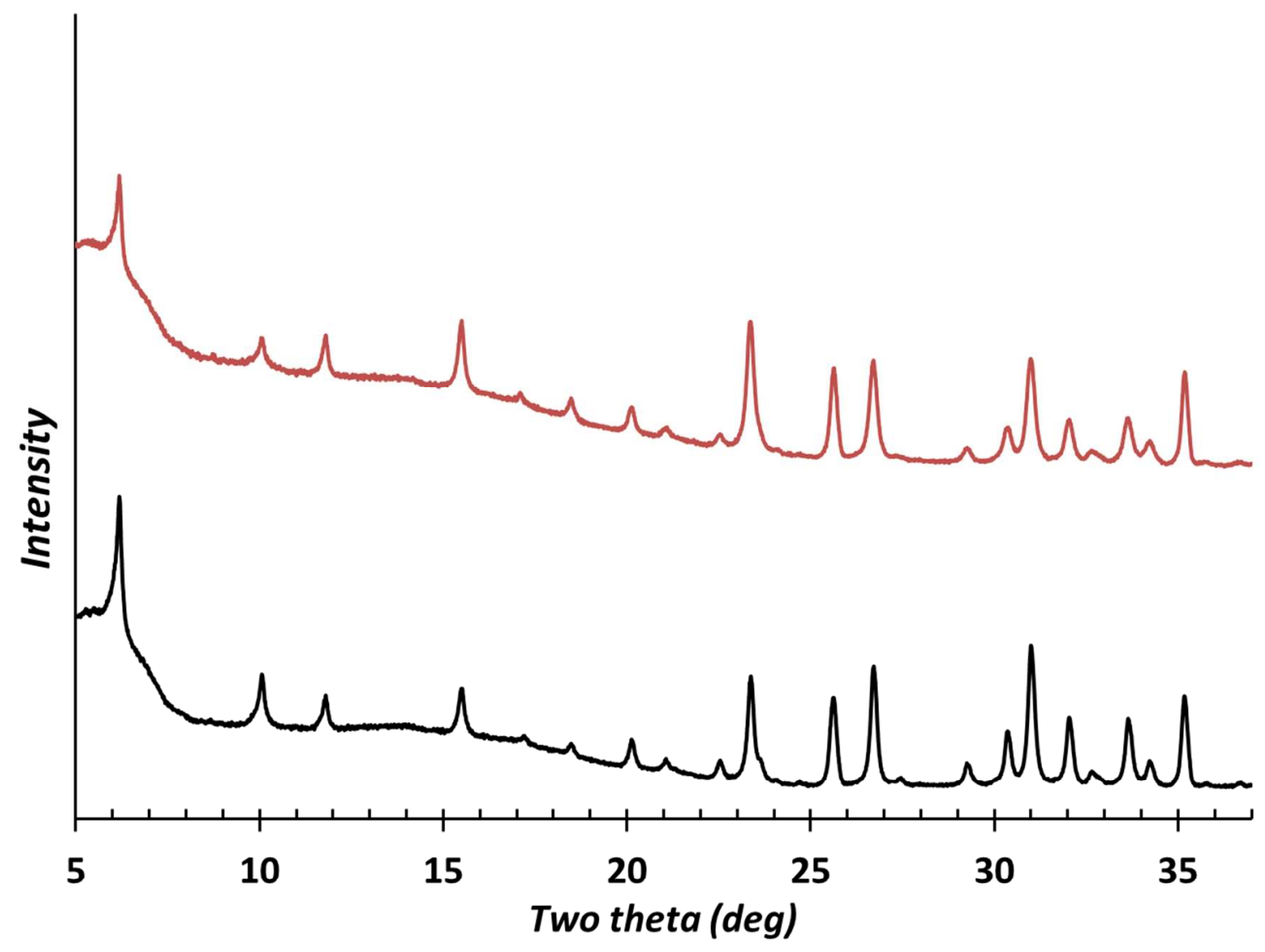

Figure S24. PXRD patterns of as-synthesized (black) and final (red) $\mathrm{NaX}$ (after 20 swings of $\mathrm{CO}_{2}$ under $\left.75 \% \mathrm{RH}\right)$. 


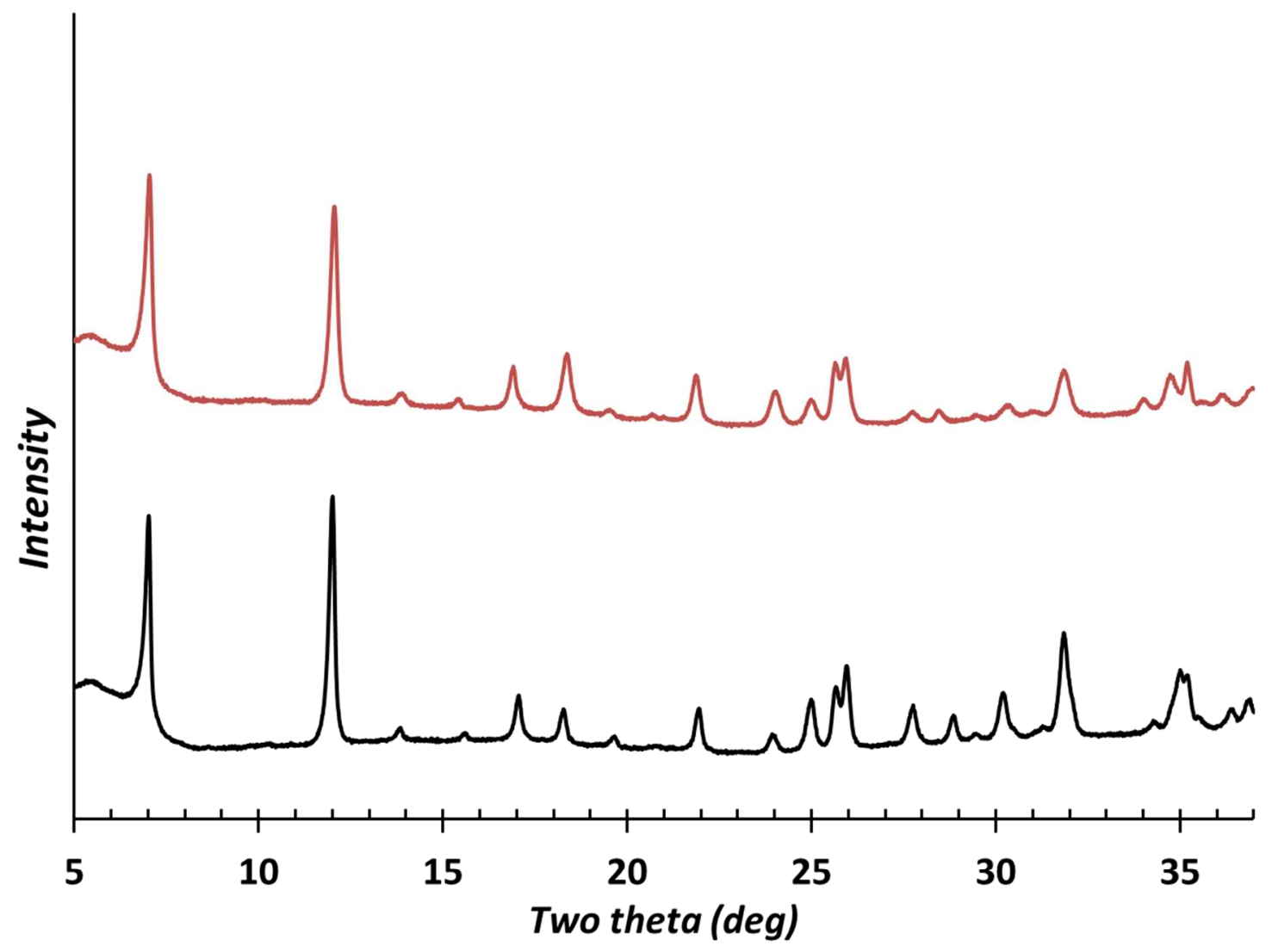

Figure S25. PXRD patterns of as-synthesized (black) and final (red) Ni-MOF74 (after 35 swings of $\mathrm{CO}_{2}$ under $75 \% \mathrm{RH}$ ). 


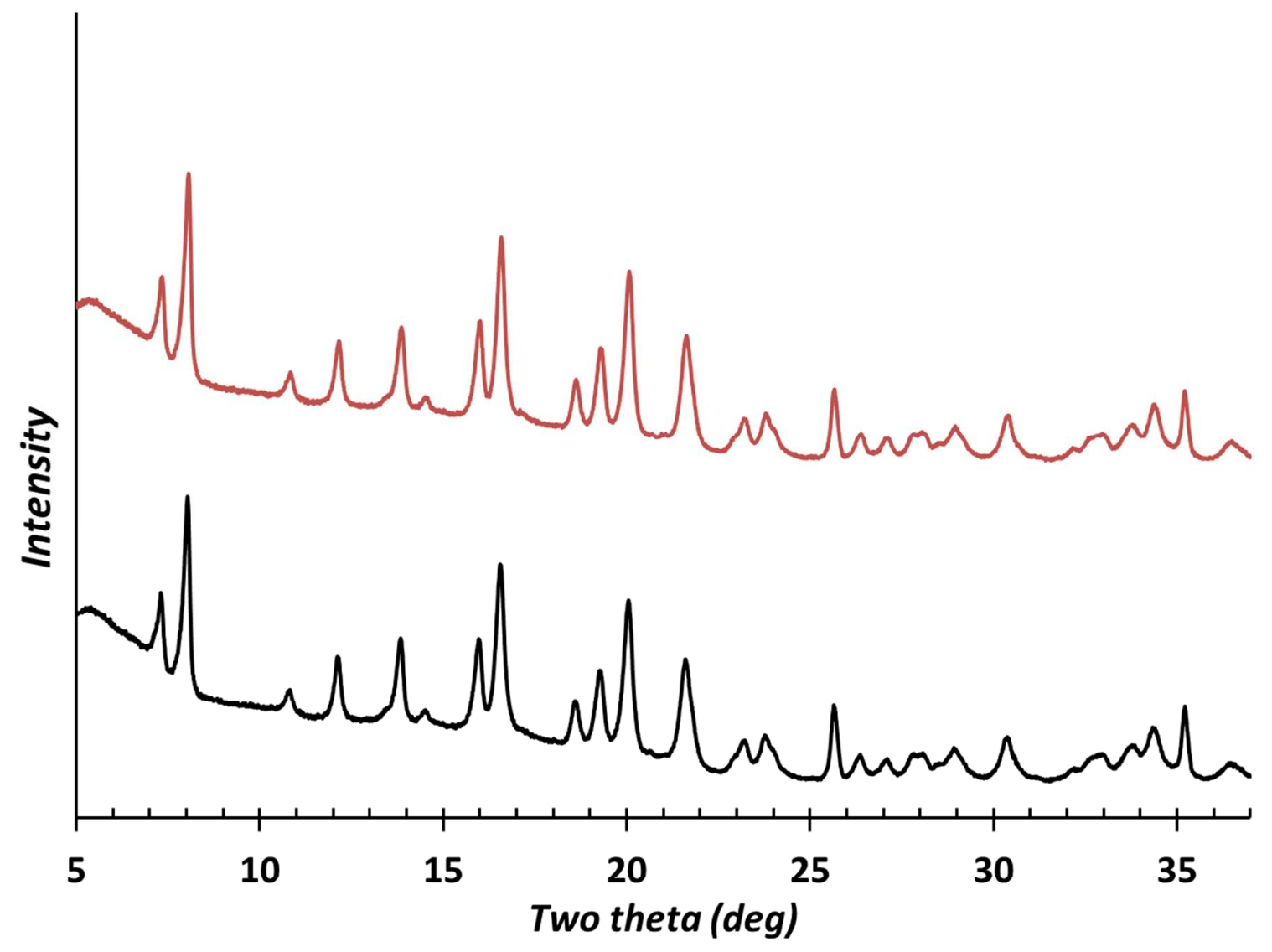

Figure S26. PXRD patterns of as-synthesized (black) and final (red) ZIF-7 (after 13 swings of $\mathrm{CO}_{2}$ under $\left.75 \% \mathrm{RH}\right)$. 